\title{
Understanding the mode-of-action of Cassia auriculata via in silico and in vivo studies towards validating it as a long term therapy for type II diabetes
}

\begin{abstract}
Ethnopharmacological relevance: Cassia auriculata (CA) is used as an antidiabetic therapy in Ayurvedic and Siddha practice. This study aimed to understand the mode-of-action of CA via combined cheminformatics and in vivo biological analysis. In particular, the effect of 10 polyphenolic constituents of CA in modulating insulin and immunoprotective pathways were studied.
\end{abstract}

Materials and methods: In silico target prediction was first employed to predict the probability of the polyphenols interacting with key protein targets related to insulin signalling, based on a model trained on known bioactivity data and chemical similarity considerations. Next, CA was investigated in in vivo studies where induced type 2 diabetic rats were treated with $\mathrm{CA}$ for 28 days and the expression levels of genes regulating insulin signalling pathway, glucose transporters of hepatic (GLUT2) and muscular (GLUT4) tissue, insulin receptor substrate (IRS), phosphorylated insulin receptor (AKT), gluconeogenesis (G6PC and PCK-1), along with inflammatory mediators genes (NF- $\kappa$ B, IL-6, IFN- $\gamma$ and TNF- $\alpha$ ) and peroxisome proliferators-activated receptor gamma (PPAR- $\gamma$ ) were determined by qPCR.

Results: In silico analysis shows that most of the top 10 enriched targets predicted for the constituents of CA are involved in insulin signalling pathways e.g. PTPN1, PCK- $\alpha$, AKT2, $\mathrm{PI} 3 \mathrm{~K}-\gamma$. Some of the predictions were supported by scientific literature such as the prediction of MAPK4 and MAPK8 for epigallocatechin gallate. Based on the in silico and in vivo findings, we hypothesized that CA may enhance glucose uptake and glucose transporter expressions via the IRS signalling pathway. This is based on AKT2 and PI3K- $\gamma$ being listed in the top 20 enriched targets. In vivo analysis shows significant increase in the expression of IRS, AKT, GLUT2 and GLUT4 CA may also affect the PPAR- $\gamma$ signalling pathway. This is based on the CA-treated groups showing significant activation of PPAR- $\gamma$ in the liver compared to control. PPAR $-\gamma$ was predicted by the in silico target prediction with high normalisation rate although it was not in the top 20 most enriched targets. CA may also be involved in the gluconeogenesis and glycogenolysis in the liver based on the downregulation of G6PC and PCK-1 genes seen in CA-treated groups. In addition, CA-treated groups also showed decreased cholesterol, triglyceride, glucose, CRP and Hb1Ac levels, and increased insulin and C-peptide levels. These findings demonstrate the insulin secretagogue and sensitizer effect of CA.

Conclusion: Based on both an in silico and in vivo analysis, we propose here that CA mediates glucose/lipid metabolism via the PI3K signalling pathway, and influence AKT thereby causing insulin secretion and insulin sensitivity in peripheral tissues. CA enhances glucose uptake and expression of glucose transporters in particular via the upregulation of GLUT2 and GLUT4. Thus, based on its ability to modulate immunometabolic pathways, CA appears as an attractive long term therapy for T2DM even at relatively low doses. 
Keyword: Cassia auriculata; Mode of action; PI3K signalling pathway; In silico target prediction; Immunometabolic pathway; PPAR- $\gamma$ 